\title{
CONTRIBUCIÓN AL CONOCIMIENTO DE LA DIETA NATURAL DE LA SERPIENTE MAZACUATA O MAZACOATL (BOA IMPERATOR DAUDIN, 1803) EN VERACRUZ, MÉXICO
}

\author{
CONTRIBUTION TO THE KNOWLEDGE OF THE NATURAL DIET OF THE MAZACOATL SNAKE (BOA IMPERATOR \\ DAUDIN, 1803) IN VERACRUZ, MÉXICO
}

\author{
Carlos Javier Pérez-Alvarado ${ }^{1}$, Felipe Agustín Lara-HernándeZ ${ }^{1}$, Víctor Vásquez-CruZ ${ }^{1 *}$ y Jair \\ PEÑA-SERRANO ${ }^{1}$ \\ ${ }^{1}$ PIMVS Herpetario Palancoatl, Avenida 19 número 5525, Colonia Nueva Esperanza, C.P. 94540, Córdoba, Veracruz, México. \\ *Correspondence: victorbiolvc@gmail.com
}

Abstract.- The snake Boa imperator is the largest species in Mexico, it is recognized by its general diet. We present the first documented case of the gray fox (Urocyon cinereargenteus) in the natural diet of the mazacuata o mazacoatl snake (B. imperator).

Keywords.- Boa, diet, Urocyon cinereoargenteus.

Resumen.- La serpiente Boa imperator es la especie de mayor tamaño en México, es reconocida por su dieta generalista. Presentamos el primer caso documentado de zorra gris (Urocyon cinereargenteus) en la dieta natural de la serpiente mazacuata o mazacoatl (B. imperator).

Palabras clave.- Boa, dieta, Urocyon cinereoargenteus.

La serpiente mazacuata o mazacoatl, Boa imperator, se distribuye en México en los estados de Sonora, Sinaloa, Nayarit, Jalisco, Colima, Michoacán, Guerrero, Oaxaca, Chiapas, San Luis Potosí, Puebla, Tamaulipas, Veracruz, Tabasco, Campeche, Yucatán y Quintana Roo, hasta el noroeste de América del Sur en Colombia (Card et al., 2016; Naturalista, 2019). Es una especie que altitudinalmente se distribuye desde el nivel del mar hasta los 1500 m s.n.m.(Heimes, 2016).

Son las serpientes de mayor tamaño en México, alcanzando una longitud total de $320 \mathrm{~cm}$ (Heimes, 2016). Es de hábitos terrestres y arborícolas. Mata por constricción y es reconocida por alimentarse de una gran variedad de presas, en los que incluye ranas (Pérez-Higareda et al., 2007), saurios (p. ej. Kartje et al., 2016), aves (p. ej. Pavón-Vázquez et al., 2016) y principalmente mamíferos pequeños y medianos (p. ej. Álvarez del Toro, 1982; Sunyer \& Galindo-Uribe, 2015). Respecto a mamíferos medianos, en la Reserva Natural Sierra Nanchititla, Estado de México, Monroy-Vilchis et al. (2011) informaron sobre un evento de depredación de un jaguarundi (Herpailurus yagouaroundi; Felidae) macho adulto que fue consumido por una Boa imperator (longitud hocico-cloaca: $273 \mathrm{~cm}$ ).
El 13 de diciembre del año 2013, alrededor de las 11:00 h, en el ejido de Caballo Blanco ( $18^{\circ} 51^{\prime} 37.99^{\prime \prime N}$; 96 $43^{\circ} 39.91^{\prime \prime O}$; WGS 84; elev. 354 m), municipio de Atoyac, Veracruz, encontramos muerto a una hembra adulta de Boa imperator (Fig. $1 \mathrm{~A}, \mathrm{LHC}=208$ $\mathrm{cm}, \mathrm{LT}=232 \mathrm{~cm}$ ) a la orilla de un sendero dentro de un parche con cultivo de caña de azúcar. El individuo mostraba cortes en el cuerpo, lo que sugiere que fue sacrificada por los lugareños. En el interior del estómago encontramos un canido adulto parcialmente digerido el cual fue determinado como zorra gris, Urocyon cinereoargenteus (Carnivora: Canidae, Fig. 1 B, C, D). El fragmento de cráneo (neurocráneo) es corto, los temporales están ásperos debajo de la cresta y sobre todo lo que se resaltan en la superficie dorsal de los procesos post orbitales es cóncava en forma de U, carácter distintivo para Urocyon cinereoargenteus (Fritzell \& Haroldson, 1982). Así como, cuenta con crestas temporales en forma de lira distintivas del género en lugar de una cresta sagital prominente en otros canidos (Hall, 1981; Armstrong et al., 2011). Debido a que los permisos de colecta no se encontraban disponibles, depositamos un cupón de fotografía en la Colección de herpetología del Museo de Historia Natural de Los Ángeles (LACM PC 2437). 


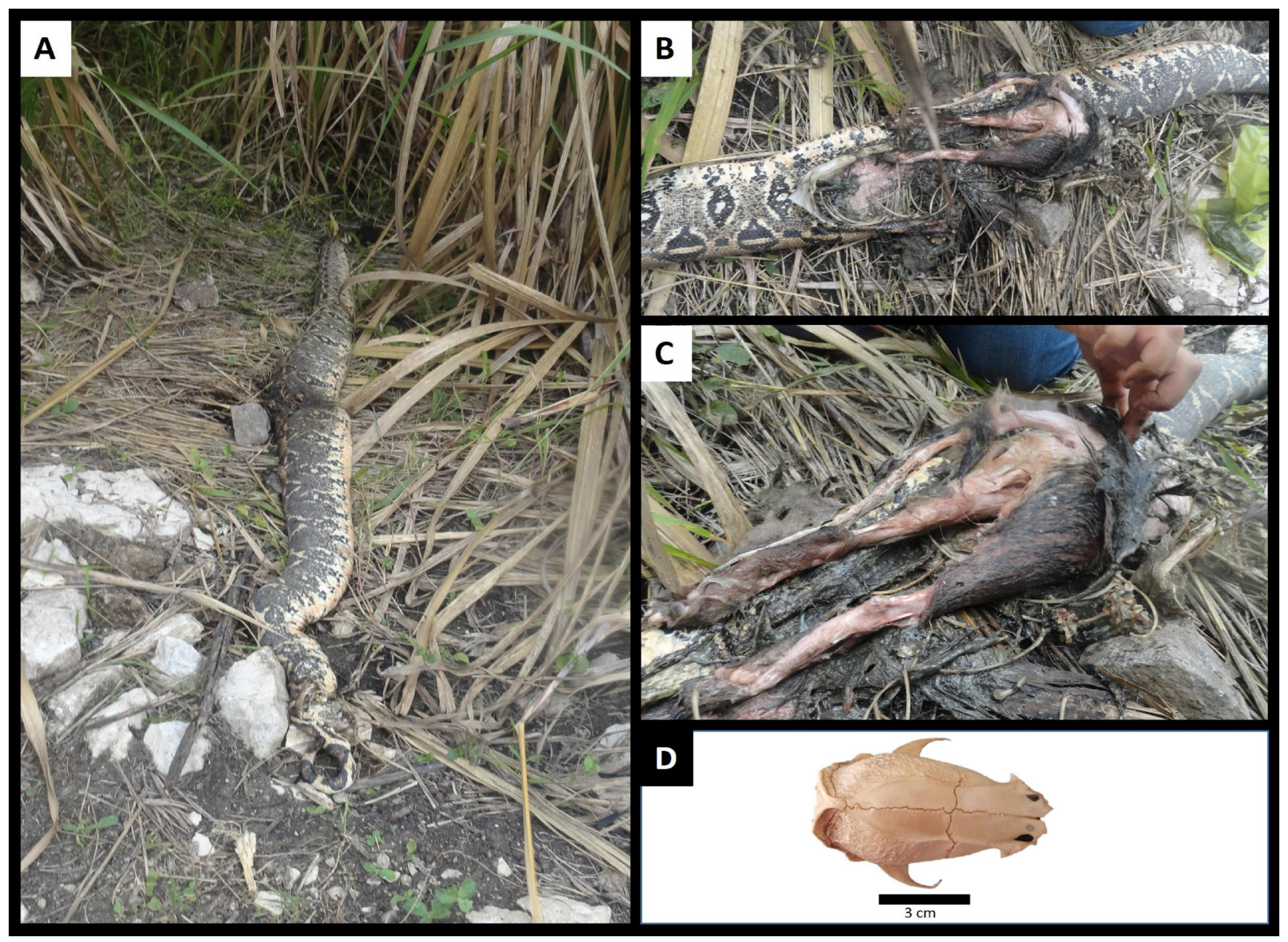

Figure 1. Dead adult female of Boa imperator from Ejido Caballo Blanco, Atoyac, Veracruz (LACM PC 2437: A). Remains of gray fox (Urocyon cinereoargenteus) in the stomach contents (B and C). Partially digested skull (occipitoparietal fragment: D).

Figura 1. Hembra adulta muerta de Boa imperator del ejido Caballo Blanco, Atoyac, Veracruz (LACM PC 2437: A). Restos de zorra gris (Urocyon cinereoargenteus) en el contenido estomacal (B y C). Cráneo parcialmente digerido (fragmento occipitoparietal: D).

Este reporte representa el primer caso documentado de $U$. cinereoargenteus, un canido, en la dieta natural de B. imperator, así como en otros miembros del género Boa (Reed \& Rodda, 2009). U. cinereoargenteus es una especie de canido común en los cultivos de caña en la zona centro de Veracruz (Gallina et al., 2016), por lo que representa una potencial e importante fuente de alimento para los individuos adultos de B. imperator.

Agradecimientos. - A la comunidad del ejido Caballo Blanco por el apoyo durante el trabajo de campo, a Ángel Méndez por ayudar en la determinación del contenido estomacal, a Tere Hernández Aguilar por la fotografía de la fig. I D, a Neftalí Camacho por catalogar la fotografía y a dos revisores anónimos por sus sugerencias para mejorar el manuscrito. .

\section{LITERATURA CITADA}

Álvarez del Toro, M. 1982. Los reptiles de Chiapas. Instituto de Historia Natural del estado de Chiapas. Tuxtla Gutiérrez, Chiapas, México. 248 pp.

Armstrong, D., J. Fitzgerald \& C. Meaney. 2011. Mammals of Colorado, Second Edition. Boulder, Colorado: University Press of Colorado. 
Card, D. C., D.R. Schield, R.H. Adams, A.B. Corbin, B.W. Perry, A.L. Andrew, G.I.M. Pasquesi, E.N. Smith, T. Jezkova, S.M. Boback, W. Booth \& T.A. Castoe. 2016. Phylogeographic and population genetic analyses reveal multiple species of Boa and independent origins of insular dwarfism. Molecular Phylogenetics and Evolution 102: 104-116.

Fritzell, E.K. \& K.J. Haroldson. 1982. Urocyon cinereoargenteus. Mammalian Species. 189.

Gallina, S., P. López-Colunga, C. Valdespino \& V. Farías. 2016. Abundancia relativa de la zorra gris Urocyon cinereoargenteus (Carnivora: Canidae) en la zona centro de Veracruz, México. Revista de Biología Tropical 64 (1): 237-249.

Hall, E.R. 1981. The mammals of North America. Second edition. Jbhn Wiley and sons. New York. 923-946.

Heimes, P. 2016. Herpetofauna Mexicana Vol. 1: Snakes of Mexico. Edition Chimaira, Frankfurt am Main, Germany.

Kartje, M.E., J.P. Corneil, C.E. Montgomery \& V.H. Reynoso. 2016. Ctenosaura oaxacana (Oaxacan Spiny-Tailed Iguana). Predation. Herpetological Review 47: 463.
Monroy-Vilchis, O., Ó. Sánchez \& V. Urios. 2011. Consumption of an adult Puma yagouaroundi (Felidae) by the snake Boa constrictor (Boidae) in Central Mexico. Revista Mexicana de Biodiversidad 82:319-321.

Naturalista. 2019. Mazacuata (Boa imperator). Comisión Nacional para el Conocimiento y uso de la Biodiversidad. Consulta 10 de mayo 2019. https:/www.naturalista.mx/taxa/539399-Boaimperator

Pavón-Vázquez, C.J., L.N. Gray, B.A. White \& A.S. Harrison. 2016. Boa imperator Daudin, 1803. Diet. Mesoamerican Herpetology 3 (2): 490-492.

Reed, R.N. \& G.H. Rodda. 2009. Giant Constrictors: Biological and Management Profiles and an Establishment Risk Assessment for Nine Large Species of Pythons, Anacondas, and the Boa constrictor. Informe de archivo abierto del estudio geológico de EE. UU. 2009-1202, Reston, Virginia. 